\title{
QUALIDADE DE VIDA DE IDOSOS COM DOENÇAS CRÔNICAS ACOMPANHADOS PELA ESTRATÉGIA SAÚDE DA FAMÍLIA
}

Ana Patricia Firmino ${ }^{1}$

Andréa Carvalho Araújo Moreira ${ }^{2}$

Francisco Wellington Dourado Júnior ${ }^{2}$

Francisca Alanny Rocha Aguiar ${ }^{3}$

Danielle Rocha do Val ${ }^{3}$

\author{
https://orcid.org/0000-0003-0113-1005 \\ https://orcid.org/0000-0001-9855-1449 \\ https://orcid.org/0000-0002-3326-338X \\ https://orcid.org/0000-0002-6281-4523 \\ https://orcid.org/0000-0002-0503-9575
}

Objetivo: Descrever a qualidade de vida de idosos com doenças crônicas, acompanhados pela Estratégia Saúde da Família. Método: Estudo descritivo e transversal, realizado com 90 idosos acompanhados pelas equipes de saúde da família de um município do Ceará, Brasil, entre outubro e novembro de 2017. Os dados foram coletados nos domicilios dos idosos utilizando um questionário do perfil social e saúde e instrumentos validados sobre qualidade de vida de idosos, WHOQOL-BREF e WHOQOL-OLD. Para análise dos dados empregou-se estatística descritiva. Resultados: A autoavaliação da qualidade de vida obteve média de 48,75. Verificou-se maior escore para a qualidade de vida de idosos nos domínios das relações sociais (57,98) e intimidade (53,04). Houve menor escore para o domínio "atividades passadas", presentes e futuras $(37,23)$ e para o domínio meio ambiente $(45,45)$, onde as facetas de maiores prejuizos foram recursos financeiros $(35,00)$ e recreação e lazer $(26,07)$. Conclusão: Idosos com doenças crônicas alcançam niveis medianos de qualidade de vida, sendo as relações sociais e intimidade os domínios que se apresentam com niveis mais satisfatórios, necessitando melhorar aspectos relacionados à assistência social e de saúde mental e física dos idosos.

Descritores: Qualidade de Vida: Idosos: Doenças Crônicas.

\section{QUALITY OF LIFE OF ELDERLY PEOPLE WITH CHRONIC DISEASES MONITORED BY THE FAMILY HEALTH STRATEGY}

Objective: To describe the quality of life of elderly people with chronic diseases monitored by the Family Health Strategy. Methods: A descriptive and cross-sectional study, carried out with 90 elderly people monitored by health teams in the city of Ceará, Brazil, between October and November 2017. Data were collected in the homes of the elderly using a questionnaire of social and health profile and validated instruments on quality of life of the elderly, WHOQOL-BREF and WHOQOL-OLD. For data analysis, descriptive statistics is used. Results: An average self-assessment of quality of life of 48.75 . There was a higher score in the domain of social relations (57.98) and a lower score in the domain of the environment (45.45) where the facets that point the most damage to the quality of life of the elderly were financial resources (35.00) and recreation and leisure (26.07). The intimacy domain used the highest score (53.04) and the past, present and future activities lowest score (37.23) domain by WHOQOL-OLD. Conclusion: Elderly people with chronic diseases reached average levels of quality of life, with social relationships and domains of intimacy presenting more satisfactory levels, needing to improve aspects related to health care and usage practices

Descriptors: Quality of Life; Elderly; Chronic Disease.

\section{CALIDAD DE VIDA DE LAS PERSONAS MAYORES CON ENFERMEDADES CRÓNICAS MONITOREADAS POR LA ESTRATEGIA DE SALUD FAMILIAR}

Objetivo: Describir la calidad de vida de las personas mayores con enfermedades crónicas monitoreadas por la Estrategia de Salud Familiar. Método: Estudio descriptivo y transversal, realizado con 90 personas mayores monitoreadas por equipos de salud en la ciudad de Ceará, Brasil, entre octubre y noviembre de 2017. Los datos fueron recolectados en los hogares de ancianos utilizando un cuestionario de perfil social y de salud e instrumentos validados en calidad de vida de los ancianos, WHOQOL-BREF y WHOQOL-OLD. Para el análisis de datos, se utiliza estadistica descriptiva. Resultados: Una autoevaluación promedio de calidad de vida de 48.75. Hubo un puntaje más alto en el dominio de las relaciones sociales (57.98) y un puntaje más bajo en el dominio del medio ambiente (45.45) donde las facetas que señalaron el mayor daño a la calidad de vida de los ancianos fueron los recursos financieros (35.00) y recreación y ocio (26.07). El dominio de la intimidad utilizó el puntaje más alto (53.04) y el dominio del puntaje más bajo de las actividades pasadas, presentes y futuras (37.23) por WHOQOL-OLD. Conclusión: Las personas mayores con enfermedades crónicas alcanzaron niveles promedio de calidad de vida, con relaciones sociales y dominios de intimidad que presentan niveles más satisfactorios, que necesitan mejorar aspectos relacionados con la atención médica y las prácticas de uso Descriptores: Calidad de Vida; Anciano; Enfermedad Crónica.

'Secretaria Municipal de Saúde. Santana do Acaraú, CE, Brasil.

${ }^{2}$ Universidade Estadual Vale do Acaraú. Sobral, CE, Brasil.

${ }^{3}$ Centro Universitário UNINTA. Sobral, CE, Brasil.

Autor correspondente: Francisco Wellington Dourado Júnior - Email: wjunior0599@gmail.com Recebido: 06/08/2020 - Aceito: 03/11/2020 


\section{INTRODUÇÃO}

O processo de envelhecimento é entendido como individual, irreversível, biológico, diversificado e variável entre os indivíduos de uma espécie, constituindo-se como um grande desafio para a saúde pública, especialmente em países em desenvolvimento, onde a pobreza, a desigualdade social e a desorganização do planejamento público são marcantes ${ }^{1}$

Neste contexto, alguns indicadores de saúde têm sofrido mudanças, o que tem justificado a necessidade de reorganização dos serviços de saúde em vista ao atendimento integral da pessoa idosa ${ }^{2}$. Assim, como reflexo do crescimento do número de idosos no Brasil, observa-se uma mudança no perfil de saúde dessa população marcado principalmente pela predominância de doenças crônicas e suas complicações. Isso se deve ao fato da própria velhice trazer riscos para um potencial desenvolvimento de comorbidades, além do estilo de vida adotado pelo indivíduo que influencia diretamente nesse processo de adoecimento ${ }^{3}$.

As Doenças Crônicas Não Transmissiveis (DNCT) que abrangem as doenças circulatórias, câncer, diabetes e obesidade caracterizam-se como multifatoriais e podem se desenvolver no decorrer da vida, causando forte impacto no âmbito da saúde pública, tendo em vista que há uma projeção de um considerável aumento de indivíduos diagnosticados com DCNT ${ }^{4}$. Desta forma, essas vêm se destacando pela morbimortalidade que acarretam, pelo alto grau de limitação das pessoas nas atividades diárias, ocasionando perda da qualidade de vida e comprometendo a capacidade funcional, em especial dos idosos ${ }^{5}$.

Dentre as condições crônicas que mais acometem a população geriátrica, a Hipertensão Arterial Sistêmica (HAS) e a Diabetes mellitus (DM) se configuram como um grande desafio para os sistemas de saúde, por afetar a vida de milhões de pessoas, e resultar em grande impacto econômico e alto custo social ${ }^{6-7}$.

No que concerne aos impactos causados pelas DCNT em idosos, a dimensão da qualidade de vida é a mais prejudicada ${ }^{5}$. Vale dizer que o conceito de Qualidade de Vida (QV) depende da condição sociocultural e das pretensões pessoais de cada um, além de estar relacionado com a autoestima e o bem-estar pessoal, os quais dependem de aspectos de ordem funcionais, psicossociais, econômicos e de saúde . $^{2}$

Acrescenta-se ainda que a OV na terceira idade pode ser compreendida como um construto multidimensional e depende da inter-relação de múltiplos fatores como saúde, atividade, autonomia, independência funcional e recursos socioeconômicos?. Para os idosos com doenças crônicas a QV se torna algo ainda mais complexo, pois viver com uma condição crônica torna mais difícil a manutenção da funcionalidade global do organismo ${ }^{10}$

No cotidiano dos serviços de saúde é importante mensurar a qualidade de vida dos idosos com doenças crônicas, pois a partir desses resultados as decisões e condutas terapêuticas dos profissionais terão um subsídio para possibilitar um cuidado direcionado a integralidade da saúde do idoso, identificando as necessidades reais e potenciais por meio de uma avaliação multidimensional ${ }^{11}$.

A atuação do enfermeiro em gerontologia requer conhecimento do processo de envelhecimento, desenvolvendo atividades por meio de um processo de cuidar para atender todas as necessidades multidimensionais do idoso, sob um olhar holístico e humanizado a fim de promover a sua qualidade de vida, em especial sua autonomia e independência funcional ${ }^{12}$.

Logo, considera-se que este estudo seja significativo para profissionais da saúde, gestores e sociedade, com o intuito de ampliar pesquisas sobre o tema, sobretudo associado a doenças que implicam em limitações nos idosos, a fim de se obter melhores resultados para a prática clínica diária e promoção da saúde, com foco na melhoria da qualidade de vida dessa população.

Frente ao exposto, o objetivo deste estudo foi descrever a qualidade de vida de idosos com doenças crônicas, acompanhados pela Estratégia Saúde da Família.

\section{MÉTODO}

\section{Tipo de Estudo}

Estudo descritivo, transversal e de abordagem quantitativa.

\section{Local do Estudo}

Estudo realizado na cidade de Morrinhos-Ceará. Segundo o Instituto Brasileiro de Geografia e Estatística (IBGE), o referido município é situado na região norte do estado do Ceará, a 208 Km de Fortaleza, com população estimada, para 2019, de 22.534 pessoas $^{13}$. Morrinhos possui, em sua zona urbana, 90 idosos cadastrados na Estratégia Saúde da Família, com diagnóstico simultâneo de HAS e DM.

\section{Participantes do Estudo}

A amostra foi constituida por 70 idosos com doenças crônicas, acompanhados e cadastrados nos territórios adscritos das Unidades Básicas de Saúde (UBS) da zona urbana, que atenderam aos seguintes critérios de inclusão: pessoas com idade igual ou superior a 60 anos, de ambos 
os sexos e diagnosticados simultaneamente com HAS e DM. Foram excluídos do estudo 20 idosos, considerando os seguintes critérios: não tiveram seus endereços encontrados durante a visita domiciliar ou por incapacidade cognitiva autodeclarada para responder ao instrumento de coleta de dados ou que foram a óbito durante o período da pesquisa.

\section{Coleta de dados}

A coleta ocorreu entre outubro e novembro do ano de 2017. O protocolo do estudo envolveu primeiramente o contato com a coordenação da Atenção Básica (AB) para apresentação da pesquisa e levantamento do quantitativo de participantes potencialmente elegíveis. Em seguida, com apoio dos Agentes Comunitários de Saúde (ACS) foi realizado o levantamento dos endereços dos participantes e programado a realização de visitas domiciliares, para aplicação dos instrumentos da coleta de dados. Os participantes foram previamente contatados pelo seu ACS, para agendamento das visitas domiciliares. Nessa ocasião foram esclarecidos sobre o Termo de Consentimento Livre e Esclarecido, a garantia do sigilo e anonimato das respostas, o direito de participar ou não voluntariamente da pesquisa, sem qualquer prejuizo.

Para a coleta de dados utilizou-se um questionário de perfil social e de saúde, elaborado pelos autores, para levantar informações acerca das variáveis relacionadas a sexo, idade, estado civil, anos de estudo, situação ocupacional, situação de moradia, arranjo familiar, atividade física, tabagismo, etilismo, uso de açúcar, uso de medicamentos e presença de complicações.

Para a mensuração da OV foram aplicados dois instrumentos elaborados pela Organização Mundial da Saúde, o WHOOOL-OLD e WHOOOL-BREF, ambos validados no Brasil. A versão em português do instrumento WHOQOL-BREF apresentou características satisfatórias de consistência interna, validade discriminante, validade de critério, validade concorrente e fidedignidade teste-reteste ${ }^{14}$. Assim como a versão brasileira do WHOQOL-OLD, que também apontou em sua validação consistência interna, medida pelo coeficiente alfa de Cronbach em cada faceta, bem como no conjunto de itens ${ }^{15}$.

O WHOQOL-BREF, instrumento genérico, está constituído por quatro domínios: físico, psicológico, relações sociais e meio ambiente. Já o WHOQOL-OLD, específico para idosos, possui seis facetas: funcionamento dos sentidos, autonomia, atividades passadas, presentes e futuras, participação social, morte e morrer e intimidade. Esses instrumentos foram utilizados em outros estudos e apre- sentaram propriedades psicométricas satisfatórias na mensuração da OV em idosos ${ }^{16,17}$.

A interpretação dos resultados dos instrumentos WHOQOL-BREF e WHOQOL-OLD foi realizada através de uma escala de Likert (de um a cinco) e, quanto maior a pontuação, melhor a qualidade de vida). Porém, no instrumento WHOQOL-BREF foi necessário recodificar os valores das questões 3, 4, $26(1=5)(2=4)(3=3)(4=2)(5=1)^{15}$. No WHOQOL-OLD foram recodificados os valores das questões 01, 02, 06, 07, 08, 09 e $10(1=5)(2=4)(3=3)(4=2)$ $(5=1)$. A conversão das questões é utilizada com o objetivo de padronizar todas as respostas do instrumento, de forma que a resposta mais positiva seja cinco e a mais negativa seja $u^{18}$. Quanto às variáveis relacionadas à qualidade de vida, os escores dos domínios do WHOQOL-BREF foram calculados através da soma dos escores da média de " $n$ " questões que compõem cada domínio. O resultado é multiplicado por quatro, sendo representado em uma escala de quatro a 20. Em seguida, os escores dos domínios são convertidos para uma escala de zero a 100, quanto mais perto de 100 melhor a OV. Ressalta-se que, no WHOQOL-BREF cada faceta é representada por uma única questão, e, por esse motivo, os escores das facetas não são calculados, são calculados somente os domínios ${ }^{19}$.

Quanto às variáveis do WHOQOL-OLD, os escores das facetas foram calculados de três maneiras diferentes. $\bigcirc$ escore bruto foi calculado a partir da soma de todas as questões pertencentes a cada uma das facetas, retornando um resultado variante entre quatro e 20 . O escore padronizado por meio da divisão do escore bruto da faceta pelo número de questões da faceta (quatro), retornando um resultado variante entre um e cinco. Em seguida, o escore foi calculado a partir da conversão do escore bruto para uma escala de zero a $100^{20}$.

\section{Procedimentos de análise e tratamento dos dados}

Os dados obtidos foram tabulados em planilhas do programa Excel, Microsoft, versão 2013, e analisados de maneira descritiva. Para as variáveis relacionadas ao perfil social e de saúde dos participantes foram calculadas as frequências absolutas e relativas.

\section{Aspectos éticos}

A pesquisa respeitou os preceitos bioéticos constantes na Resolução 466/12, do Conselho Nacional de Saúde, que regulamenta pesquisas envolvendo seres humanos. $\mathrm{O}$ estudo foi aprovado pelo Comitê de Ética e Pesquisa do Centro Universitário INTA (UNINTA), sob Parecer n으 2.338.396. 


\section{RESULTADOS}

Quanto às características sociodemográficas dos participantes do estudo, verificou-se idade média de 75,5 anos, com predominância do sexo feminino (74,2\%), sendo estes casados (50\%) e viúvos (37,1\%). A maioria (58,5\%) não tinha nenhum ano de estudo, eram aposentados (77,1\%), residindo em casa própria (91\%), em convívio com seu cônjuge (39\%) e filhos (20\%)

Sobre as principais condições de saúde dos idosos, observou-se predomínio de idosos que não realizavam atividades físicas (86\%) e que não eram tabagistas, nem etilistas (83\%). A maioria dos idosos consome adoçante (46\%) ao invés de açúcar (43\%), além de tomarem quatro ou mais medicamentos diariamente (66\%). Ainda, 73\% não referiram nenhuma complicação e 14\% tiveram Acidente Vascular Cerebral (AVC) e Infarto Agudo do Miocárdio (IAM).

Com relação aos domínios que abrangem a OV, a Figura 1 retrata as médias do WHOQOL-BREF. Os resultados demonstraram o maior escore médio para o domínio " $\mathrm{Re}$ lações sociais" $(57,98)$ e o menor escore médio para o domínio "Meio ambiente" $(45,45)$, sendo que, o valor total do escore foi 49,62 .

Figura 1 Descrição da qualidade de vida segundo domínios do WHOQOL-BREF.

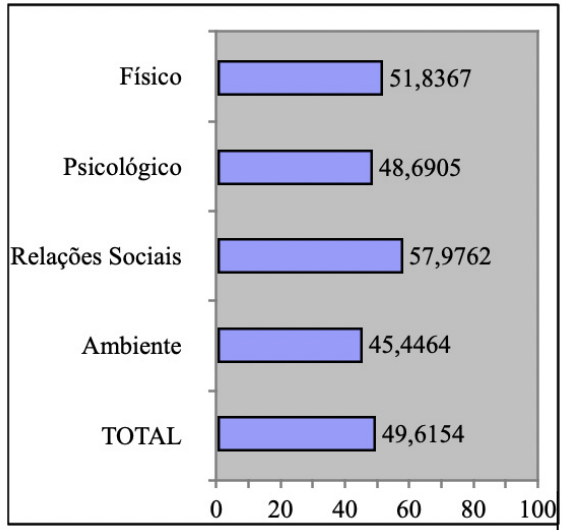

A Figura 2 exibe uma descrição da qualidade de vida segundo facetas do WHOOOL-BREF. Quanto ao domínio físico, evidenciou-se maior média para a faceta dependência de medicação ou de tratamentos (83,93). Com relação à faceta mobilidade, a média foi 54,29; na faceta sono e repouso, a média foi de 53,21; na faceta dor e desconforto, ainda avaliada pelo domínio físico, a média foi 50,00

No que se refere ao domínio psicológico, a faceta autoestima obteve melhor média $(61,07)$, seguida da faceta imagem corporal e aparência $(57,86)$.
Com relação à faceta relações pessoais, avaliada pelo domínio relações sociais, a média dos participantes foi $(63,93)$ e, quanto à faceta suporte e apoio pessoal, os idosos obtiveram média 60,71. A menor média avaliada foi na faceta atividade sexual $(49,29)$.

Na faceta cuidados de saúde, que é avaliada pelo domínio meio ambiente, a média foi 59,64 e para a faceta ambiente do lar, a média foi 57,86. Os menores índices deste domínio foram identificados nas facetas recursos financeiros $(35,00)$ e recreação e lazer $(26,07)$.

Em relação à avaliação da qualidade de vida, a auto avaliação dos idosos sobre a qualidade de vida e a satisfação com a saúde obteve uma média de $(48,75)$.

Figura 2 Descrição da qualidade de vida segundo as facetas do WHOQOL-BREF.

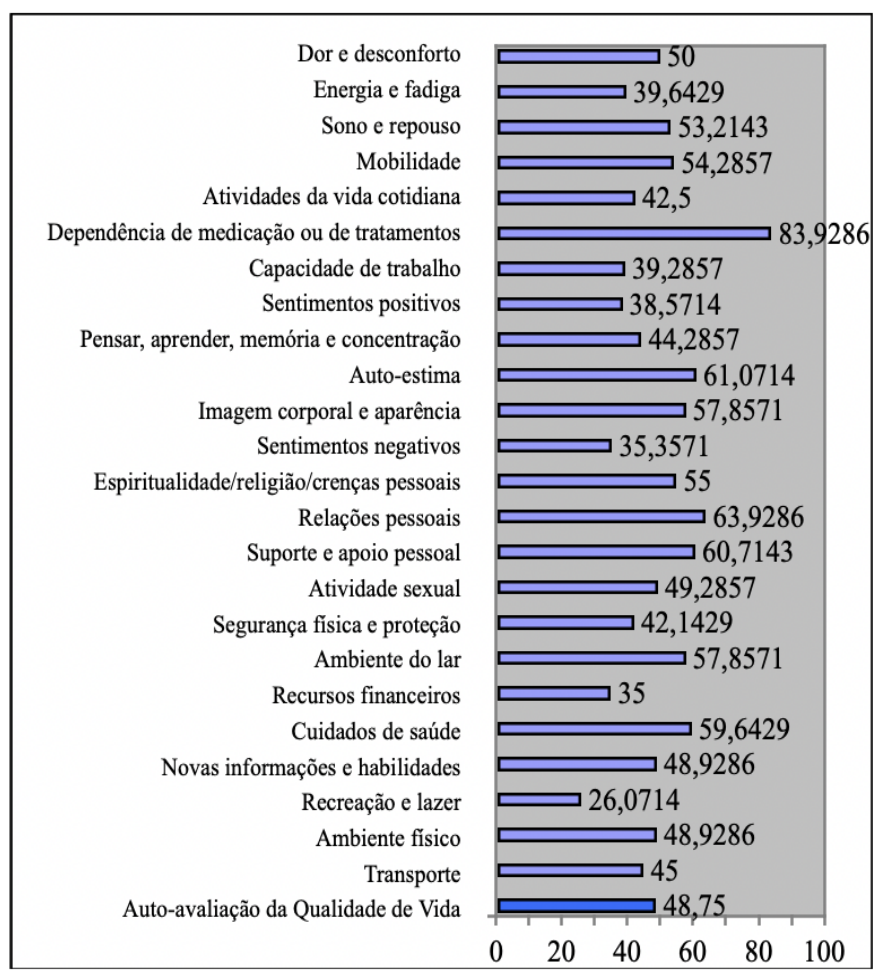

No que se refere aos dados do WHOQOL-OLD (Figura 3), o maior escore médio foi obtido na faceta "Intimidade" $(53,04)$, que avaliava os sentimentos de companheirismo, amor, oportunidades para amar e oportunidades para ser amado dos idosos. O menor escore médio obtido foi na faceta "Atividades passadas, presentes e futuras" (37,23), que avaliava a satisfação dos idosos para continuar alcançando outras realizações na vida, o quanto receberam o reconhecimento que mereciam, a satisfação com o que alcançaram e o quão feliz estavam com as coisas que podiam esperar dali para frente. O valor total do escore foi $(47,89)$, conforme mostra a Figura 3. 
Figura 3 Descrição da qualidade de vida segundo facetas do WHOQOL-OLD.

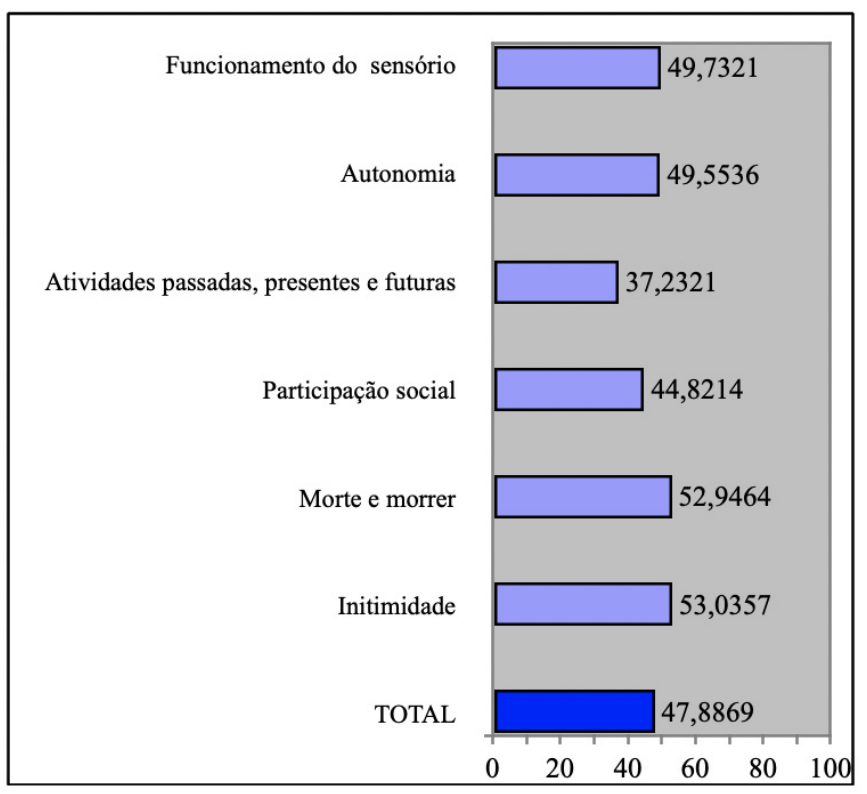

\section{DISCUSSÃO}

Participaram do estudo idosos com doenças crônicas, sendo predominantemente mulheres, assim como resultados de outros estudos ${ }^{21-22}$. As demais características sociodemográficas dos participantes, em sua maioria, casados, aposentados, com idade superior a 70 anos e analfabetos funcionais, assemelham-se aos achados de outra pesquisa realizada com um grupo de idosos de um município do Rio Grande do Sul23.

O estado civil casado pode influenciar na prevalência da satisfação na dimensão da intimidade medida pelo WHOQOL-OLD, conforme observado nos participantes desse estudo. A importância dessa variável encontra-se na solidez dos relacionamentos duradouros mantidos pelos idosos, geralmente com início na juventude e perdurando por toda transformação da vida até a velhice, além de frequentemente tornarem-se cuidadores uns dos outros, visto a saída dos filhos de casa, as mudanças biopsicossociais e o aparecimento de doenças ${ }^{24}$.

Além disso, verificou-se uma satisfação dos participantes quanto à faceta do suporte social e apoio pessoal, que pode estar associado ao fato de a maioria dos idosos morarem com o cônjuge, que geralmente é outro idoso. No entanto, isso fragiliza o autocuidado com a saúde, pois no Brasil existe a tendência do aumento do número de idosos cuidadores de outros idosos ${ }^{24}$

Os idosos da presente pesquisa auto relataram, em sua maioria, não realizar atividade física, fato que se observa também em resultados de outro estudo ${ }^{25}$. O sedentarismo pode trazer consequência como dor e desconforto, faceta que foi avaliada pelo instrumento WOOOL-BREF com média baixa. Além disso, os idosos apresentaram déficit na faceta locomoção, o que pode estar relacionado também ao sedentarismo, visto que uma pesquisa realizada em Fortaleza que avaliou a mobilidade de idosos por meio do teste Timed Up \& Go (TUG), concluiu que a prática de atividade física regular pode estar relacionada à melhor mobilidade funcional ${ }^{26}$.

No que se refere à qualidade de vida dos idosos com doenças crônicas, de acordo com o WHOQOL-BREF, o maior escore médio foi identificado no domínio "relações sociais", isso significa que, para esses idosos, a participação em atividades do cotidiano na comunidade, a tomada de decisão preservada e a atividade sexual tiveram maior impacto na qualidade de vida. Essa realidade pode estar relacionada às atividades realizadas pelos enfermeiros das unidades de saúde que realizam acompanhamento contínuo aos idosos com HAS e DM, articulam grupos de promoção da saúde, organizam atividades de lazer, momentos de interação social, a fim de promover a participação dos idosos em grupos sociais e apoiá-los na busca da OV na velhice ${ }^{20}$.

Nesse estudo, o menor escore médio obtido no WHOQOL-BREF foi identificado no domínio "meio ambiente", que conglomera fatores relacionados a segurança física e proteção, ambiente no lar, recursos financeiros, cuidados de saúde e sociais. Tais fatores inserem-se no contexto multidimensional da velhice, que exige um planejamento financeiro por parte dos indivíduos para esse momento da vida, tendo em vista os riscos presentes de condições desfavoráveis à OV, dificuldades no acesso a medicações, propensão à ocorrência de quedas ou até mesmo a possibilidade do abandono familiar. Ademais, faz-se necessário também o desenvolvimento de políticas públicas voltadas para assistirem as necessidades das populações em vulnerabilidade social ${ }^{27}$.

Essa realidade condiz com outro estudo realizado em Minas Gerais, que encontrou baixo nível no mesmo domínio, fato este que pode estar relacionado às dificuldades dos idosos na adaptação ao local que vivem, com renda inferior a três salários mínimos, isolamento social e diminuição do grau de autonomia desse grupo populacional ${ }^{28}$.

A análise da dor em idosos é fundamental para averiguar a influência no modo de vida desse público, visto os prejuízos causados e a limitação física desencadeada pela dor. Neste estudo, os idosos apresentaram limitação física por queixas dolorosas, o que desperta preocupação e exige uma atenção especial. Pesquisadores defendem que a dor interfere na percepção que cada pessoa tem de sua vida, 
portanto, requer ações adequadas visando o bem-estar e controle para redução das queixas a fim de melhorar a capacidade funcional e sua $\mathrm{OV}^{29}$

Outro aspecto importante, o sono e repouso, foi avaliado negativamente, mostrando que o descanso ruim pode influenciar na OV desses indivíduos, pois uma qualidade do sono ruim acarreta em um aumento de cochilos durante o dia, dificuldade de concentração, supressão do sistema imunológico, diminuição da capacidade funcional que torna o idoso mais propenso a quedas e pode colaborar até mesmo para um gradativo declínio cognitivo ${ }^{30}$

Além disso, mais da metade dos participantes da pesquisa consideram-se insatisfeitos quanto às suas condições de saúde. Um estudo realizado em Montes Claros (MG), que objetivou identificar como idosos atendidos em um centro ambulatorial de referência se auto avaliavam em relação à saúde, também encontrou em seus resultados uma insatisfação por parte dos idosos em relação à saúde, utilizando como justificativa fatores como aparecimento de incapacidades físicas causadas pelas DCNT, a perda da autonomia na realização de suas atividades e queixas frequentes de dor, que geram não só desconfortos físicos, mas prejuízos emocionais e psicológicos que interferem negativamente na OV dessa população ${ }^{31}$

Quanto à avaliação da qualidade de vida através do WHOQOL-OLD, o maior escore médio identificado foi na faceta "intimidade", que corrobora com outro estudo realizado com idosos diabéticos acompanhados por uma Unidade Básica de Saúde de Brasília, que obteve também melhor média na mesma faceta. Este achado pode estar relacionado ao fato da maioria dos participantes serem casados, o que facilita os relacionamentos desses idosos ${ }^{32}$.

No WHOQOL-OLD, o menor escore médio avaliado foi na faceta "atividades passadas, presentes e futuras", corroborando com dados semelhantes de outro estudo, que atribuiu maior impacto ao grande percentual de idosos com diversas comorbidades associadas, que podem levar o indivíduo a diminuir suas perspectivas em relação ao futuro $^{33}$. Nessa faceta os baixos escores identificaram comprometimento na qualidade de vida dos idosos, onde itens como satisfação dos idosos com as conquistas no decorrer da vida e as perspectivas futuras foram avaliadas, sendo que as expectativas das pessoas variam de acordo com o seu nível sócio-econômico-cultural, o acesso aos serviços de saúde, os anseios da família e da comunidade, o nível de dependência, custo e financiamento do tratamento, entre outros $^{34}$.

Diante disso, o idoso passa a enfrentar um desafio não apenas psicológico e emocional, mas existencial, que se re- laciona ao fator da temporalidade, da relação do indivíduo com suas experiências passadas, com as adaptações exigidas pelas mudanças do presente e com a forma de olhar para o futuro, como um caminho repleto de novas possibilidades. Em meio a esse conflito interno, alguns idosos tendem à um constante declínio na sua QV e podem entrar em um processo de depressão ${ }^{12}$.

É nesse contexto que novas práticas de promoção da saúde do idoso são tão necessárias para esse público, como é o caso, por exemplo, da abordagem espiritual trabalhada principalmente em grupos coordenados por enfermeiros. O profissional de enfermagem assume papel fundamental ao considerar a religiosidade e a espiritualidade como prática inovadora do cuidado ao idoso com DCNT, de modo a assegurar um cuidado humanístico, que atenda não somente aos aspectos biológicos, mas no qual a dimensão espiritual também seja respeitada e valorizada, como estratégia de enfrentamento de estressores e meio de ressignificação da velhice ${ }^{31}$.

\section{Limitações do estudo}

Algumas limitações foram encontradas na realização da pesquisa, como: o número reduzido da amostra; o delineamento transversal, que impossibilita a análise da relação de causa e efeito de aspectos que determinam a qualidade de vida dos idosos.

\section{Contribuições para prática}

Esta pesquisa propicia subsídios aos enfermeiros, para uma prática integral do idoso com doenças crônicas, a fim de contribuir com o desenvolvimento de intervenções que visem a melhoria da OV dessa população, além de favorecer reflexões importantes acerca das necessidades geriátricas, bem como fatores que devem ser considerados na avaliação multidimensional da velhice. Os achados desta investigação podem nortear estratégias de cuidado, a nível macro e micropolítico, na assistência aos idosos de uma comunidade, com perfil similar da população estudada.

\section{CONCLUSÃO}

Os idosos com doença crônica acompanhados pela atenção primária de um município cearense alcança níveis medianos de auto percepção sobre sua qualidade de vida. Observou-se níveis maiores de qualidade de vida nos domínios relações sociais e intimidade. Por outro lado, houve pior percepção no domínio ambiente e na faceta Atividades passadas, presentes e futuras, que revela a desmotivação de perspectivas futuras na velhice.

Assim sendo, os resultados desta pesquisa trazem discussões importantes sobre preditores que podem influenciar na qualidade de vida de idosos com DCNT e suscitam reflexões acerca da importância da assistência multidi- 
mensional direcionada à população geriátrica, tendo em vista as particularidades biológicas, sociais e psicológicas do processo de envelhecimento.

Sugere-se que mais estudos procurem avaliar a qualidade de vida na velhice, sendo ampliada a capacidade de analisar, de forma mais aprofundada e robusta, os aspectos que determinam e se relacionam aos domínios da qualidade de vida.

\section{Contribuições dos autores:}

APF contribuiu na realização da coleta, análise e interpretação dos dados; ACAM na concepção e desenho do estudo, bem como na aprovação da versão final a ser publicada: FWDJ na redação e revisão crítica do manuscrito; FARA na revisão crítica do manuscrito e DRV também na revisão critica do manuscrito.

\section{REFERÊNCIAS}

01. Confortin SCSIJC, Antes DL, Cembranel F, Ono LM, Marques LP. Borges LJ, Krug R de R, D'Ors E. Condições de vida e saúde de idosos: resultados do estudo de coorte EpiFloripa Idoso. Epidemiologia e Serviços de Saúde. [Internet] 2017 [cited 2020 Apr 19]:26(2):1-13. Disponivel em: http://www.scielo.br/scielo.php?script=sci_arttextEpid=S2237-96222017000200305.

02. Sousa NFS, Medina LPB, Bastos TF, Monteiro CN, Lima MG, Barros MBA. Desigualdades sociais na prevalência de indicadores de envelhecimento ativo na população brasileira: Pesquisa Nacional de Saúde, 2013. Revista Brasileira de Epidemiologia. [Internet] 2019 [cited 2020 Apr 19]:22(2):1-13.Disponivel em: http://www.scielo.br/scielo. php?pid=S1415-790X2019000300400\&script=sci_arttext\&tlng=pt.

03. Machado WD, Gomes DF, Freitas CASL, Brito M da CC, Moreira ACA. Idosos com doenças crônicas não transmissiveis: um estudo em grupos de convivência. Revista Ciência \& Saberes-Facema. [Internet] 2017 [cited 2020 Apr 19]:3(2):445-451. Available from: http://www. facema.edu.br/ojs/index.php/ReOnFacema/article/view/194/106.

04. Diaz LR, Cruz D de ALM da. ADAPTATION MODEL INA CONTROLLED CLINICAL TRIAL INVOLVING FAMILY CAREGIVERS OF CHRONIC PATIENTS. Texto contexto - enferm. [Internet] 2017 [cited 2020 Apr 19]:26(4):e0970017. Disponivel em: http://www.scielo.br/scielo.php?script=sci_arttextEpid=S010407072017000400609\&lng=pt.

05. Becker RM, Heidemann ITSB. Promoção da saúde no cuidado às pessoas com doença crônica não transmissivel: revisão integrativa. Texto contexto - enferm. [Internet] 2020 [cited 2020 Apr 19];29:e20180250. Disponivel em: http://www.scielo.br/scielo.php?script=sci_arttext\&pid=S010407072020000100502\&lng=pt.

06. Maruthur NM, Tseng E, Hutfless S, Wilson LM, Suarez-Cuervo C, Berger Z, Chu Y, Iyoha E, Segal JB, Bolen S. Diabetes medications as monotherapy or metformin-based combination therapy for type 2 diabetes: a systematic review and meta-analysis. Annals of Internal Medicine. [Internet] 2016 [cited 2020 Apr 19];164(11):740-51. Available from: http://ojoclinico.net/sitio/wp-content/uploads/2016/05/ Maruthur-NM-Diabetes-Medications-Ann-Int-Med-2016.pdf.

7. Yusufali AM, Khatib R, Islam S, Alhabib KF, Bahonar A, Swidan HM Khammash U, Alshamiri MQ, Rangarajan S, Yusuf S. Prevalence, awareness, treatment and control of hypertension in four Middle East countries. Journal of Hypertension. [Internet] 2017 [cited 2020 Apr 19];35(7):1457-1464. Available from: https://www.ncbi.nlm.nih.gov/ pubmed/28486270.

8. Santos $L$ dos. Qualidade de vida em idosos de uma estratégia de saúde da familia em um municipio do norte gaúcho. Rio Grande do Sul. Trabalho de Conclusão de Residência: Universidade Federal da Fronteira Sul. [Internet] 2019 [cited 2020 abr 26]. Available from: https:// rd.uffs.edu.br/handle/prefix $/ 3600$

9. Molina NPFM, Tavares DM dos S, Haas VJ, Rodrigues LR. Religiosidade, espiritualidade e qualidade de vida de idosos segundo a modelagem de equação estrutural. Texto contexto - enferm. [Internet] 2020 [cited 2020 Apr 19];29:e20180468. Available from: http://www.scielo.br/scielo.php?script=sci_arttext\&pid=S010407072020000100323\&lng=en.

10. Lima BM, Araujo FA, Scattolin FAA. Qualidade de vida e independência funcional de idosos frequentadores do clube do idoso do municipio de Sorocaba. Arquivos Brasileiros de Ciências da Saúde. [Internet] 2020 [cited 2020 Apr 19];41(3):168-175. Available from: https://www. portalnepas.org.br/abcshs/article/view/907.

11. Borges CL, Freitas MC, Guedes MVC, Silva MJ, Leite SFP. Prática clínica do enfermeiro no cuidado ao idoso fragilizado: estudo de reflexão. Revista de Enfermagem UFPE. [Internet] 2016 [cited 2020 Apr 19];10(2):914-918. Available from: https://www.researchgate.net/ publication/299305005_NURSING_CLINICAL_PRACTICE_IN_THE_ FRAIL_ELDERLY_CARE_REFLECTION_STUDY_PRATICA_CLINICA_ DO_ENFERMEIRO_NO_CUIDADO_AO_IDOSO_FRAGILIZADO_ESTUDO_DE_REFLEXAO_PRACTICA_CLINICA_DEL_ENFERMERO_EN_ EL_CUIDADO_AL_AN

12. Ferreira WFS, Silva CMJ, Oliveira EM. A judicialização da saúde e suas dimensões na gerontologia: uma contribuição da enfermagem. Revista Saúde e Desenvolvimento. [Internet] 2017 [cited 2020 Apr 19]:11(9):1-18. Available from: https://www.uninter.com/revistasaude/ index.php/saudeDesenvolvimento/article/view/792/487

13. Instituto Brasileiro de Geografia e Estatística (IBGE). Conheça cidades e estados do Brasil. [Internet]. 2020 [cited 2020 abr 26]; Available from: https://cidades.ibge.gov.br/brasil/ce/morrinhos/panorama.

14. Fleck MP de A. O instrumento de avaliação de qualidade de vida da Organização Mundial da Saúde (WHOQOL-100): caracteristicas e perspectivas. Ciênc. saúde coletiva. [Internet] 2000 [cited 2020 abr 26]:5(1):33-38. Available from: http://www.scielo.br/scielo. php?script=sci_arttext\&pid=S141381232000000100004\&lng=en.

15. Fleck MP, Chachamovich E, Trentini C. Development and validation of the Portuguese version of the WHOQOL-OLD module. Rev. Saúde Pública. [Internet] 2006 [cited 2020 abr 26]:40(5):785-791. 
Available from: http://www.scielo.br/scielo.php?script=sci_arttextEpid=S003489102006000600007\&lng=en.

16. Manso MEG, Maresti LTP, Oliveira HSB de. Análise da qualidade de vida e fatores associados em um grupo de idosos vinculados ao setor suplementar de saúde da cidade de São Paulo, Brasil. Rev. bras. geriatr. gerontol. [Internet] 2019 [cited 2020 abr 26];22(4):el90013. Available from: http://www.scielo.br/scielo.php?script=sci_arttextEpid=S180998232019000400201\&lng=pt.

17. Grechi MS, Baptista A, Magalhães D. Mobile Application for Assessing Quality of Life in the Elderly. EasyChair Preprint. [Internet] 2020 [cited 2020 abr 26];1:1-10. Available from: https://easychair.org/ publications/preprint_open/dKRw.

18. Fleck MPA, Chachamovich E, Trentini CM. Projeto WHOQOL-OLD: método e resultados de grupos focais no Brasil. Rev. Saúde Pública. [Internet] 2003 [cited 2020 abr 26];37(6):793-799. Available from: http://www.scielo.br/scielo.php?script=sci_arttextEpid=S0034-89102003000600016\&lng=en.

19. Pedroso B, Pilatti LA, Reis DR. Cálculo dos escores e estatística descritiva do WHOQOL-100 utilizando o Microsoft Excel. Revista Brasileira de Qualidade de Vida. [Internet] 2009 [cited 2020 abr 26];1(1):23-32. Available from: https://periodicos.utfpr.edu.br/rbqv/ article/view/366

20. Pedroso B, Pilatti LA, Gutierrez GL, Santos CB, Picinin CT. Validação da sintaxe unificada para o cálculo dos escores dos instrumentos WHOQOL. Conexões Educação Fisica, Esporte e Saúde. [Internet] 2011[cited $2020 \mathrm{abr} 26] ; 9(1): 130-156$. Available from: https://periodicos.sbu.unicamp.br/ojs/index.php/conexoes/article/ view/8637717/5408

21. Lins IL, Andrade LVR. A feminização da velhice: representação e silenciamento de demandas nos processos conferencistas de mutheres e pessoas idosas. Mediações-Revista de Ciências Sociais. [Internet] 2018 [cited 2020 abr 26]:23(3):436-465. Available from: http:// www.uel.br/revistas/uel/index.php/mediacoes/article/view/34289.

22. Miyamoto AY, Cabrera EMS, Esteves M, Lourenção LG. Qualidade de vida de idosos em uma instituição de longa permanência. Arq. Ciênc. Saúde. [Internet] 2016 [cited 2020 abr 26];23(2):36-40. Available from: http://www.cienciasdasaude.famerp.br/index.php/racs/ article/download/292/192/

23. Trennepohl C, AlvesKB, SilveiraNA, Brunelli AV, ThumC, Costa DH. Condições físicas e qualidade de vida de idosos participantes de oficinas de dança da universidade aberta à terceira idade-UNATI.CATAVENTOS-Revista de Extensão da Universidade de Cruz Alta. [Internet] 2020 [cited 2020 abr 26]; 22(1): el80136. Available from: http://200.19.0.178/index.php/cataventos/article/ view/48/137.

24. Reis RD, Dias EN, Batista MA, Silva JV da. Cuidar de idosos com doença de parkison: sentimentos vivenciados pelo cuidador familiar.Enferm. Foco. [Internet] 2019 [cited 2020 abr 26]:10(5):155-160. Available from: http://revista.cofen.gov.br/index.php/enfermagem/ article/view/2294/683.
25. Brito KOD, Menezes TN de, Olinda RA de. Incapacidade funcional: condições de saúde e prática de atividade física em idosos. Rev. Bras. Enferm. [Internet] 2016 [cited 2020 abr 26]; 69(5):825-832. Available from: http://www.scielo.br/pdf/reben/v69n5/0034-7167-reben-69-05-0825.pdf.

26. Moraes MPL, Sousa IAFC, Vasconcelos TB. Relação entre a capacidade funcional e mobilidade com a prática de atividade física em idosos participantes de uma associação. Ciências \& Saúde. 2017:9(2):90-95

27. Oliveira MA, Ribeiro HF, Costa NP.Qualidade de vida de idosos amazônicos que participam de um grupo de convivência. Enferm. Foco. [Internet] 2019 [cited 2020 abr 26];10(5):26-31. Available from: http:// revista.cofen.gov.br/index.php/enfermagem/article/view/2381/629.

28. Paiva MHP, Pegorari MS, Nascimento JS, Santos AS. Fatores associados à qualidade de vida de idosos comunitários da macrorregião do Triângulo do Sul, Minas Gerais, Brasil. Ciênc. saúde coletiva . [Internet] 2016 [cited 2020 abr 26];21(11):3347-3356. Available from: http://www.scielo.br/scielo.php?script=sci_arttextEpid=S1413-81232016001103347\&lng=en.

29. Hlubocky FJ, Muffly LS, Gomez JX, Breitenbach K, Cella D, McNeer $J L$. Death anxiety, psychological distress, and quality of life (OOL) in adolescent and young adult (AYA) cancer patients with hematologic malignancies in early survivorship. J Clin Oncol. [Internet] 2016 [cited 2020 abr 26]:34(14 Suppl):10073-10078. Available from: https://ascopubs.org/doi/10.1200/JC0.2016.34.15_suppl.10073.

30. Moreno CR de C, Santos JLF, Lebrão ML, Ulhôa MA, Duarte YA de O. Problemas de sono em idosos estão associados a sexo feminino, dor e incontinência urinária. Rev. bras. epidemiol. [Internet] 2018 [cited 2020 abr 26];21(Suppl 2):el80018. Available from: http://www.scielo.br/scielo.php?script=sci_arttext\&pid=S1415790X2018000300415\&lng=en.

31. Pereira KG, Silva PLN, Oliveira MKS, Gamba MA, Alves ECS, Martins AG. Autoavaliação da saúde por idosos atendidos em um centro ambulatorial de referência. Ensino, serviços e avaliação da Atenção Básica à Saúde. [Internet] 2018 [cited 2020 abr 26]:9(5):1-29. Available from: http://www.jmphc.com.br/jmphc/article/view/434/721.

32. Lima LR, Funghetto SS, Volpe CRG, Santos WS, Funez MI, Stival MM. Qualidade de vida e o tempo do diagnóstico do diabetes mellitus em idosos. Rev. bras. geriatr. gerontol. [Internet]. 2018 Apr [cited 2020 Aug 04];21(2):176-185. Available from: http://www.scielo.br/ scielo.php?script=sci_arttextEpid=S1809-98232018000200176\&lng=en.

33. Gato JM, Zenevics LT, Madureira VSF, Silva TG, Celich KLS, Souza SS, Léo MMF. Saúde mental e qualidade de vida de pessoas idosas. Avances en Enfermeria. [Internet] 2018 [cited 2020 abr 26];36(3):302310. Available from: http://www.scielo.org.co/pdf/aven/v36n3/01214500-aven-36-03-302.pdf.

34. Ferreira LK, Meireles JFF, Ferreira MEC. Evaluation of lifestyle and quality of life in the elderly: a literature review. Rev. bras. geriatr. gerontol. [Internet] 2018 [cited 2020 abr 26];21(5):616-627. Available from: http://www.scielo.br/scielo.php?script=sci_arttextEpid=S1809-98232018000500616\&lng=en. 